\title{
SDI gives NBS a free-electron laser for basic and applied research
}

\section{Washington}

RESEARCHERS using high-intensity radiation sources will soon have an important new research tool. The National Bureau of Standards (NBS) in building a FreeElectron Laser (FEL), due to be completed in 1990, at its laboratories in Gaithersburg, Maryland. Unlike other FELs constructed so far, this will operate at wavelengths in the near-ultraviolet and visible regions as well as in the infrared, and a much higher average power. It will be used in fields ranging from atomic physics to nuclear medicine.

The $\$ 4.9$ million cost of the FEL will be borne by the Strategic Defense Initiative Organization through a contract administered by the Office of Naval Research and the Air Force Office of Scientific Research. The FEL will give its designers familiarity with the operation of free electron lasers. This knowledge could then be applied to the construction of lasers operating at even shorter wavelengths, perhaps for military purposes.

The new laser was conceived by FEL theorists at the Naval Research Laboratory as an adjunct to the $185 \mathrm{MeV}$ continuous-wave 'racetrack' electron

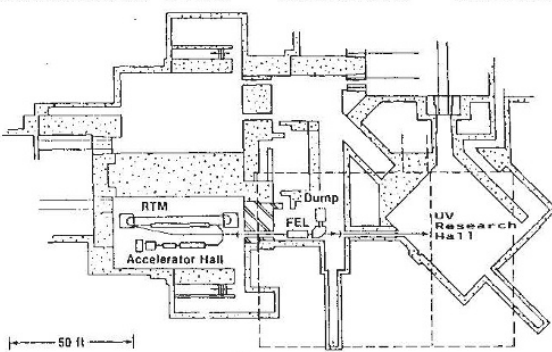

Plans of the free-electron laser and racetrack microtron.

accelerator already under construction at NBS. The accelerator acts as an energy source for the laser by providing regular pulses of electrons which, by interaction with carefully shaped irregular magnetic fields, give off energy as radiation.

According to NBS physicist Xavier Muruyama, people are "throwing away a lot of available light" with the lasers now in use. The continuous-wave accelerator, or 'microtron', will allow the new FEL to operate over a much broader spectrum of wavelengths than other free-electron lasers. Its range will extend from the $10 \mu \mathrm{m}$ infrared region to the ultraviolet region at $0.2 \mu \mathrm{m}$. Furthermore, because the repetition rate of the electron pulses feeding the laser will be essentially the $100 \mathrm{MHz}$ frequency of the microtron, the average power output of the FEL will reach hundreds or even thousands of watts.

The remarkable range of wavelengths is due both to the microtron's power and to its design. Electrons can be deflected at several points along their path through the accelerator. The full $185 \mathrm{MeV}$ of energy, corresponding to the shortest wavelength, will be achieved only when electrons pass through the entire microtron. Adjustments to the mirrors in the laser itself will be required to provide this broad range, but project director Samuel Penner considers this problem easily surmountable.

The fact that the FEL will be tunable over such a broad range of wavelengths may make it particularly suitable for medical research. For example, researchers at Harvard Medical School are using lasers in experiments on animals to break up cholesterol deposits in arteries. These experiments depend on fine-tuning the wavelength so that the laser beam destroys the blockage without hurting the artery wall. (The FEL itself will not be useful for surgery until fibre optics are develoned that can carry enough power.) In addition, the Food and Drug Administration is interested in using the FEL to test the efficacy and safety of the lasers now used in medicine.

Penner says that the fact that light pulses from the FEL will be synchronized with the pulses of synchrotron radiation from the microtron means that the FEL will be especially useful for 'pump-probe' experiments in which a pulse of light is used to excite an atom or molecule to a higher energy, and a second pulse to analyse the effects of the first. The high power provided by the laser will allow the FEL to 'read' these excitations even if it is not tuned to exactly the right wavelength. Acording to Penner, the NBS laser will be the first FEL to be used for such a variety of tasks.

\section{Planetary scientists appeal for 1990 launch of Mars Observer}

\section{Washington}

THE US Planetary Society, headed by chief spokesman and standard-bearer Carl Sagan, rallied support on Capitol Hill two weeks ago for the Mars Observer, a NASA (National Aeronautics and Space Administration) planetary mission left temporarily grounded in the aftermath of the Challenger accident. In evidence to the Senate Subcommittee on Science, Technology, and Space, Sagan stressed the importance of the Mars Observer as a step towards (ultimately manned) exploration of Mars, an objective he suggested would revitalize sagging NASA morale.

The Mars Observer, now set for a 1992 launch, was originally to leave Earth in 1990 to spend a martian year (roughly 687 Earth days) in a low Sun-synchronous orbit monitoring the magnetic fields, atmospheric circulation, gravity fields and chemical composition of Mars by remote sensing. It would also gather data on seasonal fluctuations in the parameters determining geochemical cycles and would provide clues to what happened in the planet's climatological history to dry up the once-extensive water supplies.

But the shuttle timetable until 1990 has only three slots for planetary missions, now filled by the Galileo mission to Jupiter, the Magellan radar mapper mission to Venus and the Ulysses mission to explore the polar regions of the Sun. Ulysses, a joint project with the European Space Agency (ESA), has already been delayed several times. Further delay could be acutely embarrassing to NASA.

The decision to postone the Mars Obsever launch until 1992 provoked and outcry from the planetary science community and a letter-writing campaign, sponsored by the Planetary Society, which resulted in over 20,000 letters to congressmen and NASA officials.

Delaying the launch may put the United States behind the Soviet Union in the exploration of Mars. The Soviets have recently brought forward from 1994 to 1992 plans to deploy a balloon probe to the surface of Mars, coinciding with the proposed launch date for Mars Observer. Next year, the Soviet Union will also launch a remote sensing mission of the Martian moon Phobos; former NASA Jet Propulsion Laboratory director Bruce Murray says their plan is "extremely elegant".

Sagan, Murray and Congressman George E. Brown Jr. (Democrat, California) advocate a joint programme of Mars exploration with the Soviet Union. Sagan says a national commitment to put a man on Mars would have the same effect as the Apollo lunar programme, providing a unifying goal to put NASA back on track.

The shuttle is still due to launch Mars Observer, although expendable launch vehicles, such as the Air Force Titan 34D, are acceptable alternatives. But Congressman Edward P. Boland (Democrat, Massachusetts), chairman of the House of Representatives appropriations subcommittee that oversees NASA, objects to the Mars Observer using an expendable rocket. He claims the extra $\$ 100$ million needed to launch Mars Observer in 1990 using a relatively outdated Titan $34 \mathrm{D}$ is a poor investment, considering the impending development of a more advanced Titan IV rocket schedules for 1991. But waiting until 1992 will add an additional $\$ 90-100$ million to the price. Carol Ezzell 